Check for updates

Cite this: Mater. Adv., 2020, 1,2964

Received 30th August 2020

Accepted 15th October 2020

DOI: $10.1039 / \mathrm{d} 0 \mathrm{ma} 00654 \mathrm{~h}$

rsc.li/materials-advances

\section{Delineating the enhanced efficiency of carbon nanomaterials including the hierarchical architecture of the photoanode of dye-sensitized solar cells†}

\author{
Venkatesan Srinivasan, $\ddagger^{\mathrm{a}}$ Jagadeeswari Sivanadanam, $\neq^{\mathrm{b}}$ \\ Kothandaraman Ramanujam (D) $\ddagger^{\mathrm{b}}$ and Mariadoss Asha Jhonsi (DD $\ddagger^{*^{\mathrm{a}}}$
}

\begin{abstract}
Dye-sensitized solar cells are economical and easy to fabricate in comparison to silicon-based solar cells. The visible light sensitizer dye is the main component of DSSCs, the performance of which can be enhanced if the $\mathrm{TiO}_{2}$ semiconductor is engineered to maximize the utilization of the dye together with its easy regeneration from its oxidized form. In this study, a hierarchical porous architecture was imparted to the $\mathrm{TiO}_{2}$ photoanode using size-selected (1.5 to $3 \mathrm{~nm}$ ) carbon nanomaterials (CNMs). This porous structure enhanced the accessibility of the dye to the electrolyte. Using the N719 dye as a model system, the effect of the hierarchical porous structure was demonstrated. The inclusion of CNMs together with $\mathrm{TiO}_{2}$ enhanced the short circuit current density by $31 \%$ and power conversion efficiency (PCE) by $46 \%$ compared to the CNM-free DSSCs.
\end{abstract}

\section{Introduction}

There is a continuous surge in the demand for energy due to the energy-intensive activity of human beings. Consequently, the non-replenishable sources are insufficient for the future expansion of the energy economy, which has motivated researchers to explore environmentally benign renewable energy sources. Therefore, harnessing the inexhaustible and clean energy source, solar energy, via photovoltaics occupies the central stage of renewable energy research. ${ }^{1}$ In the past two decades, significant advancement in the field of photovoltaics and efficiencies as high as $40 \%$ have been achieved by multijunction solar cell technologies. Among the various photovoltaic options, dyesensitized solar cells (DSSCs) are considered to be inexpensive due to their ease of fabrication and use of ubiquitously available materials in their fabrication. ${ }^{2}$

In DSSCs, besides the dye, wide bandgap semiconductors such as $\mathrm{TiO}_{2}$ and $\mathrm{ZnO}$ play a pivotal role in light harvesting. $\mathrm{TiO}_{2}$ offers a conducting pathway for transporting electrons from the excited state (lowest unoccupied molecular orbital

\footnotetext{
${ }^{a}$ Department of Chemistry, B. S. Abdur Rahman Crescent Institute of Science and Technology, Vandalur, Chennai - 600 048, Tamil Nadu, India. E-mail: jhonsiasha@gmail.com

${ }^{b}$ Department of Chemistry, Indian Institute of Technology Madras, Chennai-600 036, Tamil Nadu, India. E-mail: Jagadhi333@gmail.com

$\dagger$ Electronic supplementary information (ESI) available: Characterization of nanomaterial and other spectral data. See DOI: 10.1039/d0ma00654h \$ All the authors have equally contributed to this work, Dr KR facilitated writing the manuscript.
}

(LUMO)) of the dye to the conductive back contact via its conduction band (CB) and anchors sufficient dye/photosensitizers via its high surface area. Thus, energy level matching of the dye-semiconductor interface, and rationally designed semiconductor films (to reduce the loss of illuminated light and loss of injected electrons by recombination with holes, defects and crystal boundaries) are essential. A straightforward way to significantly enhance the performance of DSSCs is by manipulating the morphology and crystal phase of the semiconductor film. In the past, photoanodes were improvised using nanoarchitectures ${ }^{3-5}$ light-scattering layers, ${ }^{6,7}$ compositing with another semiconductor ${ }^{8-10}$ or graphene, ${ }^{11,12}$ doping, ${ }^{13-15}$ interfacial engineering, ${ }^{16,17}$ and TiCl $_{4}$ post-treatment methods. ${ }^{18,19}$ For instance, various nanoforms of $\mathrm{ZnO}$ were used as cascading co-sensitized nanomaterials, which enhanced the efficiency of dye-sensitized solar cells. ${ }^{20,21}$ Also, vanadium-doped $\mathrm{TiO}_{2}$ was used to fabricate the working electrode in a DSSC via the layerby-layer method, which exhibited a remarkable performance compared to the bare $\mathrm{TiO}_{2}$ particles. ${ }^{22}$

Electron transfer within $\mathrm{TiO}_{2}$ particles occurs via two phenomena, i.e. percolation through the network of particles and thermal accessibility to high energy states. Thus, to improve the electron transport, $\mathrm{TiO}_{2}$ has been explored as various nanostructures such as nanotubes, ${ }^{23}$ nanowires, ${ }^{24}$ hierarchical structures ${ }^{25}$ and specifically exposed facets. ${ }^{26}$ A film made of $\sim 20 \mathrm{~nm}$-sized $\mathrm{TiO}_{2}$ nanoparticles generally allows the semiconductor layer to achieve energy level matching and host a sufficient amount of dye. In this architecture, the electron adopts a random walk process, encountering many grain boundaries, 
trap states, and defects, which results in significant electron recombination if the interface is not well engineered. One way to achieve improved PCE is to use hierarchical spheres of $\mathrm{TiO}_{2}$ to form a secondary structure, which is concentric spherical or three-dimensional in shape. ${ }^{27}$ These materials are known for their high light-harvesting and lower electron recombination ${ }^{28,29}$ since they promote light scattering and electron transport.

Novel materials such as plasmonic metal nanoparticles (gold and silver nanoparticles), ${ }^{30}$ photonic crystals, ${ }^{31} \mathrm{WO}_{3}$-thiol-coated $\mathrm{Au}$, and nanocarbon/carbon-based nanomaterials ${ }^{32}$ have been incorporated in the fabrication DSSCs. Among the various materials composited with $\mathrm{TiO}_{2}$ in DSSC devices, carbon-based nanomaterials ${ }^{33}$ are interesting owing to their synergistic and intrinsic properties. Carbon-based nanomaterials are generally manufactured via both top-down (electrodeposition, laser ablation, exfoliation of graphite, etc.) and bottom-up approaches (solvo/hydrothermal, microwave assisted, ultrasonic synthesis, etc.). ${ }^{34,35}$ It has been reported that the addition of a certain amount of nanocarbon to $\mathrm{TiO}_{2}$ can boost the efficiency of DSSC devices. For instance, Yu et al. achieved a PCE of up to $25 \%$ by incorporating $0.75 \mathrm{wt} \%$ of graphene with $\mathrm{TiO}_{2}$ nanosheets. Carbon nanotubes were also explored for their ability to reduce the charge recombination in DSSCs. Generally, graphene is preferable over 1D carbon nanotubes since the latter has fewer intermolecular forces and connections (point contact) with $\mathrm{TiO}_{2}$. Graphene exhibits an electron mobility of $15000 \mathrm{~cm}^{2} \mathrm{~V}^{-1} \mathrm{~s}^{-1}$, which exhibits a better performance compared to that of $\mathrm{ZnO}$ (200-1000 $\left.\mathrm{cm}^{2} \mathrm{~V}^{-1} \mathrm{~s}^{-1}\right)$ and $\mathrm{TiO}_{2}\left(0.1-4 \mathrm{~cm}^{2} \mathrm{~V}^{-1} \mathrm{~s}^{-1}\right)$. According to the literature, the amount of carbon nanomaterials used is less than $1 \mathrm{wt} \%$ since loadings greater than this generally shield visible light from being harvested by the dyes, affecting the population of photogenerated electrons and PCE. Xiang et al. incorporated $0.2 \mathrm{wt} \%$ of nitrogen-reduced graphene oxide, which enhanced the PCE to $7.19 \%$ from $6.42 \%$ by circumventing electron recombination and the increasing the electron transfer efficiency. ${ }^{36}$ To alleviate the limitations associated with 1D nanomaterials, the $2 \mathrm{D}$ graphene (zero bandgap material) was explored as a bridge in a nanocrystalline semiconductor photoanode due to its lower transfer barrier, resulting in a lower recombination rate. Besides, the light-scattering effect of 2D graphene improved the short-circuit current density $\left(J_{\mathrm{sc}}\right)$ by $45 \%$ and PCE by $39 \%$ without sacrificing the open circuit voltage $\left(V_{\mathrm{oc}}\right){ }^{37} \mathrm{~A}$ DSSC with the anatase $\mathrm{TiO}_{2} @ 1.6 \mathrm{wt} \%$ reduced graphene oxide composite material prepared via a two-step hydrothermal method and stained by N719 dye showed a PCE of $7.68 \%$, which was higher than that of the pristine anatase $\mathrm{TiO}_{2}(4.78 \%) .{ }^{38}$ Chen et al. demonstrated the effect of the graphene content on device performance, and they have attained an overall PCE of $7.1 \%$ at an optimum graphene content of 2 wt $\%$, beyond which the light harvesting was affected negatively. ${ }^{39}$

Based on the literature, it can be observed that the carbon nanomaterials (CNMs) used for the modification of the $\mathrm{TiO}_{2}$ photoanode are mostly prepared via tedious methods involving several steps and high energy consumption. ${ }^{40}$ Moreover, it is necessary to understand the structure and role of CNMs in the electron collection and transport at the interface of the photoanode of a DSSC device. The effects of size-selected CNMs have rarely been investigated. To the best of our knowledge, herein, the double dialysis method is employed for the first time for selecting size-controlled CNMs ( $\sim 2 \mathrm{~nm}$-sized sheets) for application in DSSCs. In this study, different amounts of CNMs were incorporated in the photoanode to study the PCE of the resultant DSSCs. The loaded CNMs after heat treatment in air left a hierarchical pore structure, which enhanced the dye utilization and oxidized dye regeneration since the electrolyte access to the $\mathrm{TiO}_{2} /$ dye interface was enhanced.

\section{Experimental section}

The details of the materials used are presented in the ESI. $\dagger$

\section{Characterization techniques}

${ }^{1} \mathrm{H}$ NMR $(500,400 \mathrm{MHz})$ and ${ }^{13} \mathrm{C}$ NMR $(125,100 \mathrm{MHz})$ spectra of the carbonaceous nanomaterials were recorded on a Bruker NMR spectrometer in $\mathrm{D}_{2} \mathrm{O}$ with tetramethylsilane as the internal standard. Fourier-transform infrared (FTIR) spectra were recorded on a JASCO FTIR-4100 spectrometer using a KBr cell and $1064 \mathrm{~nm}$ laser. Powder X-ray diffraction (PXRD) patterns were recorded using a Bruker D8 advance diffractometer equipped with a $\mathrm{Cu} \mathrm{K} \alpha$ source $(1.5406 \AA$ ). The solution-state absorption spectra were measured on a JASCO V-650 UV-visible spectrophotometer. Diffuse reflectance UV-Vis spectra were recorded employing a JASCO model V-570 solid-state UV-Vis spectrometer. Emission spectra were measured in $\mathrm{CHCl}_{3}$ solvent using a JASCO FP-6300 spectrofluorimeter. The photovoltaic performance (current density vs. voltage, $J-V$ ) was measured under one sun condition using a LOT Technologies solar simulator equipped with a $150 \mathrm{~W}$ xenon lamp and air mass (AM) 1.5 global filter, which provided a light power of $100 \mathrm{~mW} \mathrm{~cm} \mathrm{~cm}^{-2}$. The spectral intensity of the solar simulator was chosen considering the short-circuit current from the standard silicon solar cell (Type: RC2-1010, SN: 0520160006, SPD Laboratory, Inc.). A dark mask with a circle-shaped opening in the middle was placed on the DSSC to expose the active area of the DSSC to light. Electrochemical impedance spectroscopy (EIS) measurement was performed using a Metrohm Autolab 302N potentiostat/galvanostat (AUT86242) with the FRA32M module controlled by the NOVA 1.11 software. EIS fitting was performed using the ZSimWin 3.6 software.

\section{Synthesis of CNMs}

Citric acid, sucrose and dithiobiurea in the weight ratio of $2: 2: 1$ was initially dissolved in $50 \mathrm{~mL}$ of ultrapure water by sonication for $20 \mathrm{~min}$. Then, the resulting colorless clear solution was transferred to a Teflon-lined stainless steel autoclave (100 mL capacity) and kept in a muffle furnace at $180{ }^{\circ} \mathrm{C}$ for 8 h. ${ }^{41}$ After the autoclave was cooled, the unreacted residues were removed by filtration, and the clear orange filtrate was centrifuged at $3000 \mathrm{rpm}$ for $15 \mathrm{~min}$ to remove the minute suspended particles. Further, the clear supernatant solution 
was loaded in a separating funnel and washed with dichloromethane to remove the unreacted organic moieties. Finally, it was filtered using a 0.2 micron (pore size of $200 \mathrm{~nm}$ ) cellulose syringe filter followed by volume reduction through distillation.

\section{Size-selective separation of CNMs}

The clear orange solution from the above step was dialyzed against ultrapure water using a dialysis membrane tube with a molecular weight cut off (MWCO) of $0.5-1 \mathrm{kDa}$ (pore size, $1.5 \mathrm{~nm}$ ) for $24 \mathrm{~h}$. This process allowed small organic moieties with a size of less than $1.5 \mathrm{~nm}$ (maximum of $15 \AA$ ) to exit the dialysis tube. The dialysis bath water was changed every $6 \mathrm{~h}$ to avoid the accumulation/deposition of particles inside the dialysis tube wall. After $24 \mathrm{~h}$ of dialysis, particles with a size of $>1.5 \mathrm{~nm}$ were left inside the tube. The product left inside the tube $(>1.5-200 \mathrm{~nm}$ ) was subjected to dialysis for a second time against ultrapure water in a dialysis membrane bag with an MWCO of 10-15 kDa (pore size, $3 \mathrm{~nm}$ ) for another $24 \mathrm{~h}$. The water outside turned pale yellow due to CNMs with a size ranging from 1.5 to $3 \mathrm{~nm}$ exiting the dialysis tube. This paleyellow solution was collected and evaporated in a vacuum oven to obtain dark-brown colored shining particles called CNMs. The various stages of the synthesis of the size-selective CNMs are shown in the ESI $\dagger$ (Scheme S1).

\section{Preparation of $\mathrm{CNM}$-loaded $\mathrm{TiO}_{2}$ film electrode}

The photoanode was prepared following the reported method, and the typical preparation procedure is as follows. Fluorinedoped tin dioxide coated glass plates (FTO plates) were initially cut using a diamond cutter in the dimensions of $1.5 \mathrm{~cm} \times 1.75 \mathrm{~cm}$. The plates were immersed for $15 \mathrm{~min}$ in a soap solution, ultrapure water (two times), acetone, and isopropyl alcohol under sonication. The cleaned and dried plates were treated with $40 \mathrm{mM}$ aqueous $\mathrm{TiCl}_{4}$ at $70{ }^{\circ} \mathrm{C}$ for $30 \mathrm{~min}$. The $\mathrm{TiCl}_{4}$ treatment was repeated twice, and the plates were washed with double distilled water followed by ethanol. Further, the plates were heated in a muffle furnace at $500{ }^{\circ} \mathrm{C}$ for $30 \mathrm{~min}$ and allowed to cool to room temperature naturally. The $\mathrm{TiCl}_{4}$ treated conducting side of FTO was coated with transparent nano-titania paste loaded with various amounts of CNMs $(5 \mathrm{mg}$ of CNMs was dispersed in $5 \mathrm{~mL}$ of methanol and $0.1,0.25,0.5$, $0.75,1$ and $1.5 \mathrm{~mL}$ of methanol dispersion was added to $100 \mathrm{mg}$ titania paste and stirred for $12 \mathrm{~h}$ under ambient conditions) via the doctor blade technique using a mask with a circular hole. The thickness of the transparent layer was around 5-6 $\mu \mathrm{m}$, and the coated area was $0.196 \mathrm{~cm}^{2}$. The $\mathrm{TiO}_{2}$-coated plates were heated at $500{ }^{\circ} \mathrm{C}$ for $30 \mathrm{~min}$. Upon cooling the plate, a scattering layer of $\mathrm{TiO}_{2}$ was applied (Ti-nanoxide R/SP, Solaronix) via the doctor blade technique followed by heating at $400{ }^{\circ} \mathrm{C}$ for $20 \mathrm{~min}$.

\section{Preparation of N719-dye/CNM-loaded $\mathrm{TiO}_{2}$ photoanode (FTO/ $\mathrm{TiO}_{2}$-CNMs-N719)}

FTO/TiO ${ }_{2}$ incorporated with different amounts of CNMs $(0,0.1,0.25$, $0.5,0.75,1$ and $1.5 \mathrm{wt} \%$ ) was immersed in N719 dye solution for $12 \mathrm{~h}$ $(0.5 \mathrm{mM}$ in $1: 1(\mathrm{v} / \mathrm{v})$ ratio of acetonitrile and tertiary butanol solvent mixture) to ensure maximum uptake of dye by $\mathrm{TiO}_{2}$.

\section{DSSC fabrication with $\mathrm{FTO} / \mathrm{TiO}_{2}-\mathrm{CNMs}-\mathrm{N} 719$ for $J-V$ and EIS measurements}

The FTO/TiO ${ }_{2}$-CNMs-N719 photoanode and Pt counter electrode were assembled into a sandwich-type cell with the help of a $60 \mu \mathrm{m}$ hot-melt gasket frame (MS004610-10). ${ }^{42}$ Then the cell was filled with electrolyte through a hole left intentionally open at the back of the counter electrode. The liquid electrolyte consisted of LiI (0.1 M), I 2 (0.05 M), $t$-butylpyridine (0.5 M) and 1-butyl-3methyl imidazolium iodide $(0.6 \mathrm{M})$ in a mixture of valeronitrile (15\%) and acetonitrile (85\%). A schematic representation of the fabricated device is shown in Scheme S2 (ESI $\dagger$ ).

\section{Results and discussion}

Citric acid was used as a carbon source for the graphene core, and the surrounding functional moieties of the CNMs were derived from sucrose and the presence of $\mathrm{N}$ and $\mathrm{S}$ heteroatoms from dithiobiurea, which increased the optical and electrochemical performances. Moreover, the precursor materials used for the synthesis of CNMs are easily accessible and inexpensive. The hydrothermal synthetic method employed yielded CNMs with a size of less than $3 \mathrm{~nm}$ with $\mathrm{S}$ and $\mathrm{N}$ functional groups tethered to them. The proposed mechanism for the formation of CNMs is shown in Scheme 1. Initially, dehydration occurs, followed by various processes, including hydrothermal degradation, polymerization and carbonization, leading to the nucleation and growth of CNMs. The structure of CNMs consists of two or three layers of nanometer-sized staked sheets of graphene covered with an amorphous shell bearing various functional moieties. ${ }^{35,43}$

The SEM image (Fig. 1a) on a scale of $500 \mathrm{~nm}(60000 \times$ magnification) shows the transparent layered structure of CNMs. The energy dispersive X-ray spectrum (Fig. S1a, ESI $\dagger$ ) confirms the presence of carbon, oxygen, nitrogen and sulphur. The elements originated from the precursors, and their atomic percentage is given in Fig. S1a (ESI $\dagger$ ) (inset). HR-SEM was used
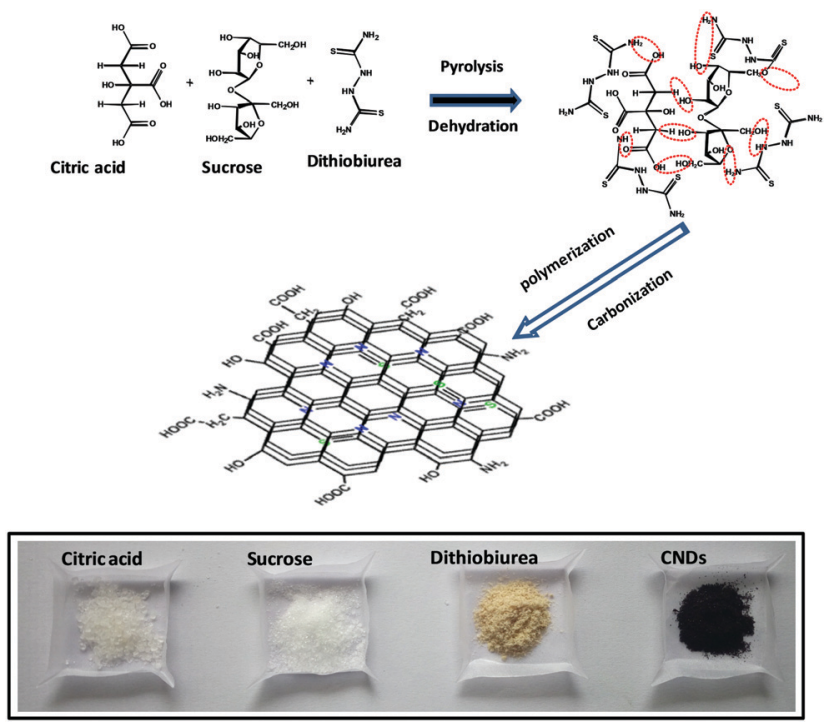

Scheme 1 Proposed mechanism for the synthesis of CNMs. 
to understand the surface morphology of the photoanode fabricated with and without CNMs, and the images are shown in Fig. 2. It can be observed that the surface of the film is smooth for $\mathrm{TiO}_{2}$, and for $\mathrm{TiO}_{2}$ with $0.5 \mathrm{wt} \%$ loading of CNMs, a few holes and surface cracks can be observed (Fig. 2a and b, respectively). This may due to the degassing of the surface functional groups of CNMs while heating the $\mathrm{TiO}_{2}$ films at $500{ }^{\circ} \mathrm{C}$. EDAX did not detect any $\mathrm{N}$ and shows a trace of $\mathrm{S}(0.8 \%$, Table S1, ESI $\dagger$ ). Therefore, to understand the presence of carbon, CHNOS analysis was performed (Table S2, ESI $\dagger$ ). The CHNOS analysis confirmed that only a trace amount of $\mathrm{N}$ and $\mathrm{S}$ was present. Therefore, most of the CNMs were incorporated into the photoanode, which were degassed during the annealing step, forming a porous $\mathrm{TiO}_{2}$ film. This probably enhanced the light scattering, similar to the hierarchical spheres of $\mathrm{TiO}_{2}$ forming a second structure that is concentric spherical or threedimensional in shape. ${ }^{44}$

The three-dimensional shape and exact particle size details derived from the HR-TEM measurements are shown in Fig. 1b. The transparent layered structure of CNMs was confirmed through the HR-TEM micrographs, as shown in Fig. S1b (ESI $\dagger$ ) (50 nm scale, $500000 \times$ magnification). The presence of particles with a size of 1.5 to $3 \mathrm{~nm}$ is discernible (Fig. 1b) from the particle size histogram (Fig. 1d). The presence of lattice fringes with spacings of $0.23 \mathrm{~nm}$ and $0.31 \mathrm{~nm}$ are possibly related to the (100) and (101) planes of CNMs, respectively (Fig. 1c). Further, the fast Fourier transform (inset of Fig. 1d) image shows the crystalline nature of the prepared CNMs. Atomic force microscopy (AFM) measurement gave the surface roughness with topographic details in non-contact mode (Fig. S1c, ESI $\dagger$ ). It indicated the layered quasi-spherical sheet structure of CNMs. The root mean square roughness value obtained from
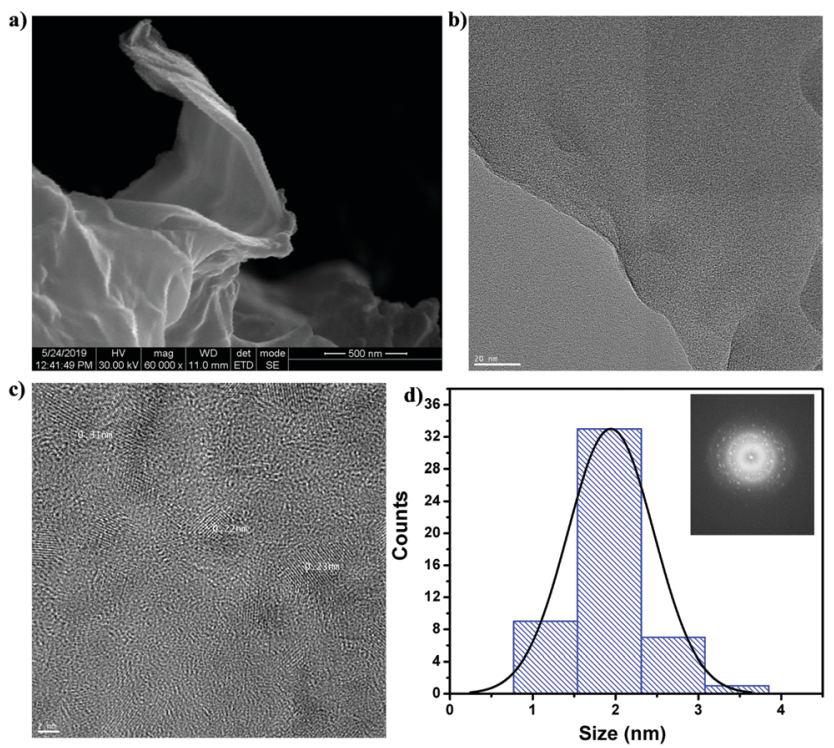

Fig. 1 (a) HR-SEM image (at 60k magnification), (b) HR-TEM image (20 nm scale) of CNMs, (c) HR-TEM image (2 nm scale) of CNMs, and (d) particle size histogram (inset FFT image) derived from the image of (b).
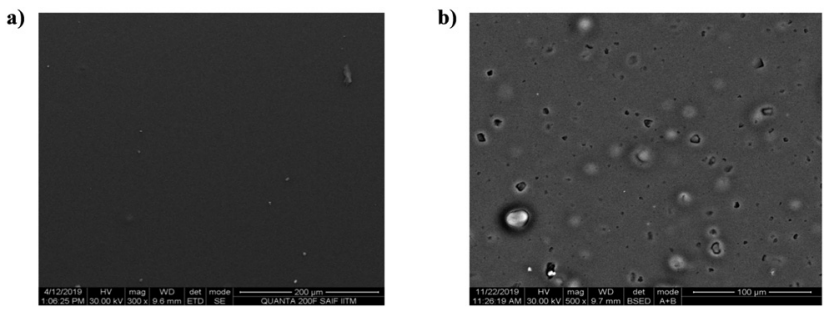

c)
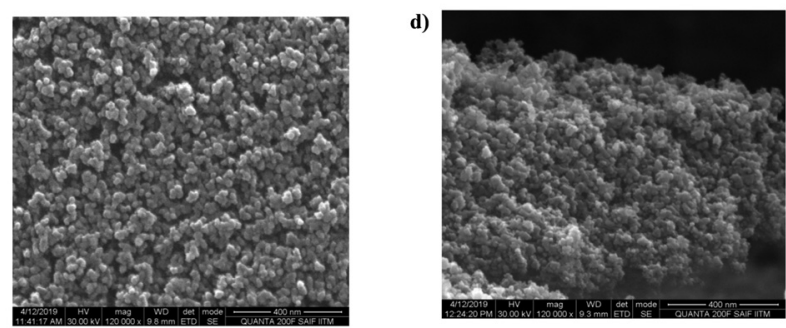

Fig. 2 HR-SEM analysis of the bare transparent $\mathrm{TiO}_{2}$ film (a and c) and $\mathrm{TiO}_{2}$ with 0.5 wt\% of CNMs (b and d).

the AFM measurement is $1.85 \mathrm{~nm}$, indicating the smooth surface of the prepared CNMs.

AFM was also used to analyze the topographical details of the $\mathrm{TiO}_{2}$ photoanode prepared with and without CNMs (Fig. S2a and b (ESI $\dagger$ ), respectively), and it was observed that the roughness for the bare $\mathrm{TiO}_{2}$ was around $8.3 \mathrm{~nm}$, which increased to $14.60 \mathrm{~nm}$ upon loading with $0.5 \mathrm{wt} \%$ of CNMs. Profilometry is a useful tool to understand the surface roughness of a material and thickness of hierarchical porous architectures (thickness of the transparent $\mathrm{TiO}_{2}$ layer is around 5-6 $\mu \mathrm{m}$, and the coated area is $0.196 \mathrm{~cm}^{2}$ ). Thus, profilometry was performed for the bare $\mathrm{TiO}_{2}$ photoanode and $\mathrm{TiO}_{2}$ loaded with 0.5 wt\% CNMs. The thickness of the bare $\mathrm{TiO}_{2}$ and $\mathrm{TiO}_{2}$ loaded with 0.5 wt $\%$ CNMs is $9.6 \mu \mathrm{m}$ and $8.5 \mu \mathrm{m}$, respectively. The average roughness of the $\mathrm{TiO}_{2}$ photoanode in the absence of CNMs is $234.36 \mathrm{~nm}$, which increased in the presence of $0.5 \mathrm{wt} \%$ of CNMs to $462.14 \mathrm{~nm}$. According to the profilometry images, it can be seen that the bare $\mathrm{TiO}_{2}$ film has a smooth surface (Fig. S3a, ESI $\dagger$ ) compared to that of the film loaded with CNMs. Moreover, the film loaded with CNM exhibits pores (Fig. S3b, ESI $\dagger$ ) developed by the burning of CNMs during the annealing step. The AFM and HR-SEM results also validate the presence of pores, as discussed earlier. The BET surface area of $\mathrm{CNM}, \mathrm{TiO}_{2}$, and 0.5 wt\% CNM-incorporated $\mathrm{TiO}_{2}$ is $2.31,64.76$, and $53.07 \mathrm{~m}^{2} \mathrm{~g}^{-1}$, respectively. The $\mathrm{N}_{2}$ isotherms and pore size distribution analysis are shown in Fig. S4a and b (ESI $\dagger)$, respectively. The pore size distribution analysis (Table S3, ESI $\dagger$ ) indicates the widening of the pore volume from 0.32 to $0.45 \mathrm{~cm}^{3} \mathrm{~g}^{-1}$ and average pore size from 13.43 to $25.31 \mathrm{~nm}$ upon the incorporation of CNMs into $\mathrm{TiO}_{2}$. The degassing of CNMs during annealing could have widened the pores, which consequently reduced the surface area, as mentioned above.

Elemental composition and functional group details of CNMs and CNM-incorporated photoanode

The elemental analysis of CNMs confirmed the presence of $40.84 \%$ carbon, $4.72 \%$ hydrogen, $10.70 \%$ nitrogen, $9.99 \%$ sulphur 
and $36.61 \%$ oxygen (Table S2, ESI $\dagger$ ), which originated from the precursors used in the synthetic process. The FT-IR spectrum of CNMs (Fig. S5a, ESI $\dagger$ ) demonstrates presence of $-\mathrm{C}-\mathrm{H},-\mathrm{NH}$, $-\mathrm{C}-\mathrm{OH},-\mathrm{NH}-\mathrm{CO}$, and $-\mathrm{SO}_{3} \mathrm{H}$ stretching vibrations at $3035 \mathrm{~cm}^{-1}$, $3129 \mathrm{~cm}^{-1}, 1166 \mathrm{~cm}^{-1}, 1404 \mathrm{~cm}^{-1}$ and $620 \mathrm{~cm}^{-1}$, respectively. The FT-IR spectrum of $\mathrm{TiO}_{2}$ indicates peaks at $\sim 3406 \mathrm{~cm}^{-1}$ due to the $-\mathrm{OH}$ stretching vibrations of the surface functional groups. The peaks below $1000 \mathrm{~cm}^{-1}$ are due to the various vibration modes of $\mathrm{Ti}-\mathrm{O}-\mathrm{Ti}$. $\mathrm{TiO}_{2}$ with $0.5 \mathrm{wt} \% \mathrm{CNMs}$ showed new peaks at $1660 \mathrm{~cm}^{-1}$, corresponding to the $\mathrm{C}=\mathrm{C}$ and $\mathrm{C}=\mathrm{O}$ stretches originating from CNMs. The ${ }^{1} \mathrm{H}$ NMR spectrum (Fig. S5b, ESI $\dagger$ ) shows signals in both the down and upfield area, confirming the presence of both aliphatic and aromatic groups. For instance, the signals in the high shielding area from 2.75-3.2 ppm are consistent with the aliphatic protons. The signals in the region above 6.5-8.2 ppm correspond to the aromatic protons of the carbon sheets and carboxylic functional moieties. The middle-range signals of 5-6.5 ppm are attributed to the protons of the various functional groups such as hydroxyl, amino, and epoxy groups. Besides, the ${ }^{13} \mathrm{C}$ NMR (Fig. S5c, ESI $\dagger$ ) spectrum shows signals in three regions, namely 40-50 ppm, which is assigned to $\mathrm{sp}^{3}$ carbon atoms attached with $-\mathrm{OH}$ groups, 70-80 ppm, which is associated with the ether linkages, and the peaks above 180 ppm assigned to the carbonyl carbon $(-\mathrm{C}=\mathrm{O})$. The results from the NMR analysis are consistent with the previous reports on carbonaceous nanomaterials. ${ }^{45}$ The thermal stability of the prepared CNMs was analyzed via thermogravimetric measurement in an air atmosphere at a temperature ramping rate of $10{ }^{\circ} \mathrm{C} \min ^{-1}$ (Fig. S5d, ESI $\dagger$ ). The observed weight loss may be due to the degradation of the amorphous shell of CNMs. The PXRD (Fig. S6, ESI $\dagger$ ) pattern of CNMs demonstrates the amorphous nature of CNMs, where a broad peak corresponding to the (002) plane was observed at $20-24^{\circ}$.

\section{Photophysical properties}

The UV-visible absorption spectrum (Fig. S7a, ESI $\dagger$ ) of CNMs exhibits two peaks, one at $\lambda_{\max }=280 \mathrm{~nm}$ and other at $352 \mathrm{~nm}$, where the former is assigned to the $\pi-\pi^{*}$ electronic transition from the $\mathrm{C}=\mathrm{C}$ bonding, and the latter to the $\mathrm{n}-\pi^{*}$ transition from the heteroatoms present on the surface. The photoluminescence was measured to study the electron-hole recombination and surface defects on the surface of CNMs (Fig. S7a, ESI $\dagger$ ). CNMs showed an emission at $540 \mathrm{~nm}$ at the fixed excitation of $360 \mathrm{~nm}$. To check the dependence of the emission on the excitation wavelength, the emission was monitored at various excitation wavelengths in the range of 300-480 $\mathrm{nm}$ at intervals of $20 \mathrm{~nm}$, which showed that the prepared CNMs exhibit excitation-dependent emission behaviour (Fig. S7b, ESI $\dagger$ ). This is probably due to the presence of various surface trap states and emissive states. Further, the fluorescence decay monitored at a wavelength of $540 \mathrm{~nm}$ (excitation of $365 \mathrm{~nm}$ ). The emission decay profile of CNMs was fitted with a multi-exponential decay (Fig. S7c, ESI $\dagger$ ) with an average lifetime of 4 ns. This provides evidence for the presence of various emissive states. ${ }^{45}$ For better understanding of the emission behaviour of CNMs, the excitation and emission contour map of CNMs in a $3 \mathrm{D}$ view is illustrated in Fig. S8a and b (ESI $\dagger$ ), which indicate the multicolor photoluminescence characteristics of CNMs. The photoexcitation of carbonaceous nanomaterials is due to $\pi$-plasmon absorption from the core carbon nanoparticles. ${ }^{46}$ Fig. S9 (ESI $\dagger$ ) shows the absorption spectra of the neat $\mathrm{TiO}_{2}$ and CNM-loaded $\mathrm{TiO}_{2}(0.5$ and $1 \mathrm{wt} \%)$ measured in the solid state, clearly indicating the scattering of light by the CNM-loaded $\mathrm{TiO}_{2}$ in the visible region. This increase in scattering is probably due to additional pores generated in the photoanode by CNMs. Therefore, employing CNMs can affect the amount of light available for the excitation of the dye. The surface coverage of the N719 dye on the hierarchical porous architecture was also investigated. Typically, high surface coverage can be achieved when the surface area of $\mathrm{TiO}_{2}$ is large. However, we observed a different trend, where the dye loading decreased with an increase in the content of CNMs (Fig. S10, ESI $\dagger$ ). This may due to the porous nature of the $\mathrm{TiO}_{2}$ surface due to the incorporation of CNMs, which may reduce the anchoring of more dye molecules on $\mathrm{TiO}_{2}$. Thus, the hierarchical porous architecture can help avoid aggregation between the dye molecules on the surface, and consequently a high PCE can be achieved. Thus, the optimization of CNMs is required to enhance the PCE of DSSCs. ${ }^{47,48}$

\section{$J-V$ measurements of DSSCs}

Photoanodes prepared with 0, 0.1, 0.25, 0.5, 0.75, 1, and $1.5 \mathrm{wt} \%$ of CNMs were assembled into DSSCs (Scheme S2, ESI $\dagger$ ) and subjected to $J-V$ measurements, and their performance is compared in Fig. 3a.

Table 1 shows the different parameters calculated from the $J-V$ characteristics including the $J_{\mathrm{sc}}, V_{\mathrm{oc}}, \mathrm{FF}$ (fill factor), and PCE. With a loading of up to $0.5 \mathrm{wt} \%$, the PCE increased, beyond which it decreased, probably due to light absorption inhibited by excessive CNMs in the photoanode. The PCE of photoanode without CNMs was $7.01 \%$, which increased to $10.24 \%$ for the photoanode loaded with $0.5 \mathrm{wt} \%$ of CNMs (almost $46 \%$ of enhancement in PCE upon the incorporation of CNMs). The decrease in the PCE and $J_{\mathrm{sc}}$ with a higher loading of CNMs may be due to the over-accumulation of CNMs on the $\mathrm{TiO}_{2}$ surface, promoting back-electron transfer. There was no apparent variation in the $V_{\mathrm{oc}}$ and $\mathrm{FF}$ measured for the various loadings of CNMs, and the PCE was mostly maintained with $J_{\text {sc }}$ controlled, which is dependent on the electron injection ability

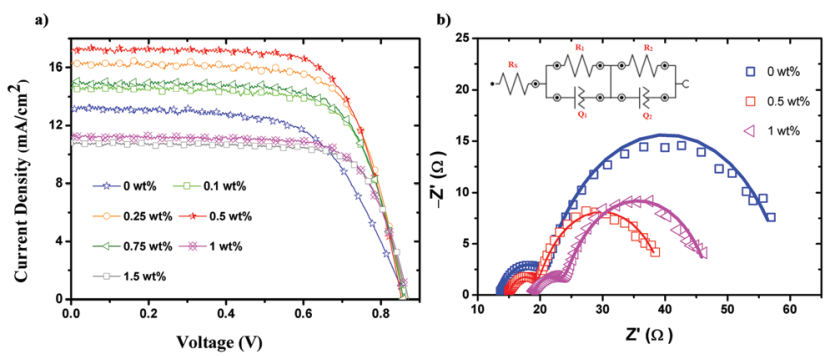

Fig. 3 (a) $J-V$ plots of DSSCs having different contents of CNMs in their photoanodes and (b) Nyquist plot obtained at 1 sun condition at $V_{\text {oc }}$ for the photoanodes loaded with 0, 0.5 and 1 wt $\%$ of CNMs (solid lines are the fit to the data). 
Table 1 Photovoltaic parameters measured under 1 sun condition from DSSCs fabricated with different loadings of CNMs

\begin{tabular}{llllrc}
\hline & & & & & Avg. $\eta$ for \\
CNMs $(\mathrm{wt} \%)$ & $J_{\text {sc }}\left(\mathrm{mA} \mathrm{cm}^{-2}\right)$ & $V_{\text {oc }}(\mathrm{V})$ & FF & $\eta(\%)$ & 3 cells $(\%)$ \\
\hline 0 & 13.10 & 0.864 & 0.61 & 7.01 & 7.01 \\
0.1 & 14.56 & 0.860 & 0.70 & 8.84 & 8.25 \\
0.25 & 16.23 & 0.858 & 0.70 & 9.85 & 9.74 \\
0.5 & 17.18 & 0.852 & 0.69 & 10.24 & 10.15 \\
0.75 & 14.94 & 0.858 & 0.70 & 8.90 & 8.53 \\
1 & 11.24 & 0.858 & 0.72 & 7.01 & 6.98 \\
1.5 & 10.82 & 0.873 & 0.72 & 6.87 & 6.55
\end{tabular}

of the dye into the $\mathrm{CB}$ of $\mathrm{TiO}_{2}$ and the charge collection efficiency of $\mathrm{TiO}_{2}$. Table S4 (ESI $\dagger$ ) shows a comparison of the literature reports on CNM-based DSSCs. To the best of our knowledge, we did not find any work incorporating sizecontrolled CNMs to enhance the PCE of DSSCs.

\section{Electrochemical impedance analysis}

The Nyquist plot shows two semicircles, where the one in the high-frequency region corresponds to the charge transfer at the counter electrode/electrolyte interface. The second semicircle in the mid-frequency region is due to the photoanode/electrolyte interface. The equivalent circuit chosen to fit the data is shown in the inset of Fig. $3 \mathrm{~b}$, which is $R_{\mathrm{s}}\left(R_{1} Q_{1}\right)\left(R_{2} Q_{2}\right)$, where $R_{\mathrm{s}}$, $R_{1}$, and $R_{2}$ are the solution-cum-contact resistance and charge transfer resistance at the counter electrode/electrolyte and photoanode/electrolyte interfaces, respectively. $Q_{1}$ and $Q_{2}$ are the constant phase element associated with $R_{1}$ and $R_{2}$, respectively. Table S5 (ESI $\dagger$ ) summarizes all the passive elements estimated through the fitting for the CNM loadings of $0,0.5$ and $1 \mathrm{wt} \% . R_{\mathrm{S}}$ increased with an increase in the CNM loading since they modified the $\mathrm{TiO}_{2}$ film, resulting in contact resistance with FTO. $R_{1}$, the charge transfer resistance across the counter electrode-electrolyte interface, was $7,4.8$ and $5.2 \mathrm{~W}$ for the CNM loading of 0, 0.5 and $1 \mathrm{wt} \%$, respectively. Considering the large error in the assumption $\left(Y_{1}\right)$ associated with $Q_{1}$, which is a constant phase element associated with $R_{1}$, for practical purposes, we can assume that $R_{1}$ is nearly the same. In contrast, $R_{2}$, which is measured across photoanode/electrolyte interface, significantly decreased from 39.0 to $20.5 \mathrm{~W}$ when the CNM loading was enhanced from 0 to $0.5 \mathrm{wt} \%$ and $23.46 \mathrm{~W}$ when the loading was increased to $1 \mathrm{wt} \%$. Since these values were measured at one sun condition and $V_{\text {oc }}$ applying $10 \mathrm{mV}$ of sinusoidal AC perturbation, the equilibrium concentration of electrons in the $\mathrm{CB}$ of $\mathrm{TiO}_{2}$ is a function of exciton recombination, and back electron transfer to the oxidized dye, making the net current across the photoanode/electrolyte interface zero. The applied alternative current perturbation modulates the current across the photoanode/electrolyte interface, which captures the charge transfer resistance associated with the electron transfer from the photoanode to $\mathrm{I}_{3}{ }^{-}$in the electrolyte and/or electron transfer from $\mathrm{I}^{-}$to the oxidized dye in the photoanode. In our study, we observed a decrease in $R_{2}$ and an increase in PCE at a loading of $0.5 \mathrm{wt} \%$ CNMs compared to the CNM-free photoanode. Therefore, the reduction in $R_{2}$ is due to the effective dye regeneration by $\mathrm{I}^{-}$in the electrolyte.
As observed from HR-SEM analysis, the size and amount of pores produced due to the degassing of CNMs were optimum at the CNM loading of $0.5 \mathrm{wt} \%$, making it easier for the electrolyte to access the oxidized dyes present in the pores of the $\mathrm{TiO}_{2}$ layer. The pores produced by the CNMs were bigger than the inherent pore size of the $\mathrm{TiO}_{2}$ film; therefore, the incorporation of CNMs is concluded to create a hierarchical pore structure, improving the performance of the DSSCs.

\section{Conclusions}

CNMs in the size range of 1.5 to $3.5 \mathrm{~nm}$ were incorporated with the mesoporous $\mathrm{TiO}_{2}$ semiconductor in the photoanode of a DSSC. To the best of our knowledge, this is the first report using size-selected CNMs in the photoanode of a DSSC. The SEM analysis demonstrated the formation of wide pores and cracks in the photoanode upon the incorporation of CNMs. These pores may be beneficial for the free flow of the electrolyte into the photoanode, reducing the overpotential related to the regeneration of the oxidized dye. This could be the reason for the improved $J_{\mathrm{sc}}$ observed. For the optimized $0.5 \mathrm{wt} \%$ CNM-loaded photoanode, the $J_{\mathrm{sc}}$ increased from $13.10 \mathrm{~mA} \mathrm{~cm}^{-2}$ to $17.18 \mathrm{~mA} \mathrm{~cm}^{-2}$.

\section{Conflicts of interest}

There are no conflicts to declare.

\section{Acknowledgements}

M. A. J. acknowledges SERB for the TARE project (Ref.: TAR/ 2018/00048, Dt: 18.02.2019). M. A. J. also acknowledges SAIF and Department of Chemistry, IIT Madras for analytical measurements. V. S. thanks to the Department of Chemistry and BSACIST for the Senior Research Fellowship. J. S. thanks for fellowship received through SERB-N-PDF (PDF/2016/002201) and DST/TMD/ SERI/HUB/1(C). Authors acknowledge the funding through DST/ TMD/SERI/HUB/1(C) for establishing DSEHC (DST-IITM solar energy harnessing center). M. A. J. acknowledges Mr Ganapathirao Kandregula, research student of Prof. KR group for the assistance in impedance fitting. M. A. J. also acknowledges to Department of Metallurgical and Materials Engineering for Titan 80-300 TEM facility.

\section{References}

1 N. Kannan and D. Vakeesan, Renewable Sustainable Energy Rev., 2016, 62, 1092.

2 K. Fan, J. Yu and W. Ho, Mater. Horiz., 2017, 4, 319-344.

3 Q. Zheng, H. Kang, J. Yun, J. Lee, J. H. Park and S. Baik, ACS Nano, 2011, 5, 5088-5093.

4 C.-J. Lin, W.-Y. Yu and S.-H. Chien, J. Mater. Chem., 2010, 20, 1073-1077.

5 Y. J. Kim, M. H. Lee, H. J. Kim, G. Lim, Y. S. Choi, N.-G. Park, K. Kim and W. Lee, Adv. Mater., 2009, 21, 3668-3673. 
6 Y.-C. Park, Y.-J. Chang, B.-G. Kum, E.-H. Kong, J. Y. Son, Y. S. Kwon, T. Park and H. M. Jang, J. Mater. Chem., 2011, 21, 9582-9586.

7 J. Lin, Y.-U. Heo, A. Nattestad, Y. Yamauchi, S. X. Dou and J. H. Kim, Electrochim. Acta, 2015, 153, 393-398.

8 Y. Li, H. Wang, Q. Feng, G. Zhou and Z.-S. Wang, Energy Environ. Sci., 2013, 6, 2156-2165.

9 X. Dang, J. Qi, M. T. Klug, P. Y. Chen, D. S. Yun, N. X. Fang, P. T. Hammond and A. M. Belcher, Nano Lett., 2013, 13, 637-642.

10 H. Choi, W. T. Chen and P. V. Kamat, ACS Nano, 2012, 6, 4418-4427.

11 N. Yang, J. Zhai, D. Wang, Y. Chen and L. Jiang, ACS Nano, 2010, 4, 887-894.

12 L. Chen, Y. Zhou, W. Tu, Z. Li, C. Bao, H. Dai, T. Yu, J. Liuand and Z. Zou, Nanoscale, 2013, 5, 3481-3485.

13 T. Ma, M. Akiyama, E. Abe and I. Imai, Nano Lett., 2005, 5, 2543-2547.

14 K. Mahmood and S. B. Park, J. Mater. Chem. A, 2013, 1, 4826-4835.

15 H. Tao, W. Chen, J. Wang, W. Ke, J. Wan, J. Wu and G. Fang, Electrochim. Acta, 2014, 137, 17-25.

16 A. K. Chandiran, M. K. Nazeeruddin and M. Grätzel, Adv. Funct. Mater., 2014, 24, 1615-1623.

17 H. Elbohy, A. Thapa, P. Poudel, N. Adhikary, S. Venkatesan and Q. Qiao, Nano Energy, 2015, 13, 368-375.

18 S.-W. Lee, K.-S. Ahn, K. Zhu, N. R. Neale and A. J. Frank, J. Phys. Chem. C, 2012, 116, 21285-21290.

19 A. Lamberti, A. Sacco, S. Bianco, D. Manfredi, F. Cappelluti, S. Hernandez, M. Quaglio and C. Pirri, Phys. Chem. Chem. Phys., 2013, 15, 2596-2602.

20 Y. Li, Z. Liu, C. Liu, J. Ya, W. Zhao, Q. Chen and J. Bai, J. Am. Ceram. Soc., 2012, 95, 1343-1347.

21 Z. Liu, Y. Li, C. Liu, J. Ya, W. Zhao, D. Zhao and L. An, ACS Appl. Mater. Interfaces, 2011, 3, 1721-1725.

22 Z. Liu, Y. Li, C. Liu, J. Ya, W. Zhao, E. Lei, D. Zhao and L. An, Solid State Sci., 2011, 13, 1354-1359.

23 L.-L. Li, C.-Y. Tsai, H.-P. Wu, C.-C. Chen and E. W.-G. Diau, J. Mater. Chem., 2010, 20, 2753-2758.

24 W.-Q. Wu, Y.-F. Xu, C.-Y. Su and D.-B. Kuang, Energy Environ. Sci., 2014, 7, 644-649.

25 F. Sauvage, D. Chen, P. Comte, F. Huang, L.-P. Heiniger, Y.-B. Cheng, R. A. Caruso and M. Graetzel, ACS Nano, 2010, 4, 4420-4425.

26 X. Tao, P. Ruan, X. Zhang, H. Sun and X. Zhou, Nanoscale, 2015, 7, 3539-3547.
27 X. Li, J. Yu and M. Jaroniec, Chem. Soc. Rev., 2016, 45, 2603-2636.

28 H.-Y. Chen, D.-B. Kuang and C.-Y. Su, J. Mater. Chem., 2012, 22, 15475-15489.

29 Q. Zhang and G. Cao, J. Mater. Chem., 2011, 21, 6769-6774. 30 S. D. Standridge, G. C. Schatz and J. T. Hupp, J. Am. Chem. Soc., 2009, 131, 8407-8409.

31 V. D. Dao, P. Kim, S. Baek, L. L. Larina, K. Yong, R. Ryoo, S. H. Ko and H. S. Choi, Carbon, 2016, 96, 139-144.

32 S. Kushwaha, S. Mandal, S. Subramanian, S. Aryasomayajul and K. Ramanujam, ChemistrySelect, 2016, 1, 6179-6187.

33 S. Paulo, E. Palomaresand and E. M. Ferrero, Nanomaterials, 2016, 6, 157-176.

34 S. Thulasi, A. Kathiravan and M. Asha Jhonsi, ACS Omega, 2020, 5, 7025-7031.

35 W. Youfu and H. Aiguo, J. Mater. Chem. C, 2014, 2, 6921-6939.

36 Z. Xiang, X. Zhou, G. Wan, G. Zhang and D. Cao, ACS Sustainable Chem. Eng., 2014, 5, 1234-1240.

37 N. Yand, J. Zhai, D. Wang, Y. Chen and L. Jiang, ACS Nano, 2010, 4, 887-894.

38 G. Cheng, M. S. Akhtar, O. B. Yang and F. J. Stadler, ACS Appl. Mater. Interfaces, 2013, 5(14), 6635-6642.

39 L. Chen, Y. Zhou, W. Tu, Z. Li, C. Bao, H. Dai, T. Yu, J. Liuac and Z. Zou, Nanoscale, 2013, 5, 3481.

40 X. Yan, X. Cui, B. Li and L. S. Li, Nano Lett., 2010, 10, 1869-1873.

41 S. Paulo, E. Palomares and E. M. Ferrero, Nanomaterials, 2016, 6(9), 157.

42 X. ang, T. Ma, G. Guan, M. Akiyama, T. Kida and E. Abe, J. Electroanal. Chem., 2004, 570, 257-263.

43 S. Dong, X. Yuqing, Z. Weimin, Z. Hongtao, Z. Ying, Q. Xiting, M. Yanfeng, Y. Yong and C. Yongsheng, J. Power Sources, 2018, 384, 328-333.

44 J.-Y. Liao, J.-W. He, H. Xu, D.-B. Kuang and C.-Y. Su, J. Mater. Chem., 2012, 22, 7910-7918.

45 V. Srinivasan, M. Asha Jhonsi, M. Kathiresan and A. Kathiravan, ACS Omega, 2018, 3, 9096-9104.

46 X. Fang, M. Li, K. Guo, J. Li, M. P. Lihua, B. M. Luoshan and X. Z. Zhao, Electrochim. Acta, 2014, 137, 634-638.

47 L. Chen, Y. Zhou, W. Tu, Z. Li, C. Bao, H. Dai, T. Yu, J. Liuac and Z. Zou, Nanoscale, 2013, 5, 3481-3485.

48 K. A. S. Fernando, S. Sahu, Y. Liu, W. K. Lewis, E. A. Guliants, A. Jafariyan, P. Wang, C. E. Bunker and Y.-P. Sun, ACS Appl. Mater. Interfaces, 2015, 7, 8363-8376. 